\title{
A Case Series of Malignant Otitis Externa Mimicking Malignancy
}

\author{
Vengathajalam Selvamalar ${ }^{1}$, Nik Adilah Nik Othman ${ }^{1,2, *}$, Mohd Khairi Md Daud ${ }^{1,2}$
}

\section{ABSTRACT}

Malignant otitis externa is an inflammation of the external auditory canal with preceding osteomyelitis of the temporal bone and the adjacent structures that could be potentially lethal. Malignant otitis externa may present with cranial nerve involvements and massive spread of disease mimicking nasopharyngeal carcinoma or any other malignancies on imaging. Two elderly patients who presented with severe otalgia and significant facial nerve palsy and lower cranial nerve palsies showing extensive spread of disease are reported in this case series. They both had resolution of disease after a prolonged course of antibiotics and cortical mastoidectomy for disease clearance in one of them.

\section{KEYWORDS}

malignant otitis externa; Pseudomonas aeruginosa; osteomyelitis; cranial nerves palsies

\section{AUTHOR AFFILIATIONS}

${ }^{1}$ Department of Otorhinolaryngology-Head and Neck Surgery, School of Medical Sciences, Health Campus, University Sains Malaysia, Kubang Kerian, Kelantan, Malaysia

2 Hospital University Sains Malaysia, Health Campus, Kubang Kerian, 16150, Kelantan, Malaysia

* Corresponding author: School of Medical Sciences, Health Campus, University Sains Malaysia, Kubang Kerian, 16150, Kelantan, Malaysia; e-mail: adilahkk@usm.my

Received: 27 June 2020

Accepted: 5 November 2020

Published online: 14 April 2021

Acta Medica (Hradec Králové) 2021; 64(1): 36-41

https://doi.org/10.14712/18059694.2021.6

(c) 2021 The Authors. This is an open-access article distributed under the terms of the Creative Commons Attribution License (http://creativecommons.org/licenses/by/4.0), which permits unrestricted use, distribution, and reproduction in any medium, provided the original author and source are credited. 


\section{INTRODUCTION}

Malignant otitis externa (MOE) is an aggressive infection of the external ear canal and temporal bone. MOE was first reported by Toulmouche in 1838 (1). Another name for MOE is necrotizing otitis externa or osteomyelitis of the temporal bone. Patients with MOE usually presents with painful ear discharge with hearing loss, tinnitus and throbbing headaches that is not responding to medical treatment. The ear canal is edematous with granulation tissue at the osteocartilaginous junction on otoscopic examination. The usual causative organism is Pseudomonas aeruginosa and the infection can be lethal with extension of the infection to the skull base and central nervous system causing cranial nerve palsies, meningitis and brain abscesses. The commonly involved cranial nerve is the facial nerve as it exits the stylomastoid foramen, subsequently the glossopharyngeal, vagus and accessory nerves as they exit the jugular foramen and the hypoglossal nerve as it exits the hypoglossal canal. In petrous apex involvement, the trigeminal and abducens nerve can be affected and in cavernous sinus and clivus involvement, the olfactory, troclear and abducens nerve may be affected as well. We report two cases of malignant otitis externa in two elderly female patients presented with lower cranial nerve palsies and extensive spread of disease described on computed tomography (CT).

\section{CASE REPORT 1}

This patient is a 50-year-old lady who presented with dysphagia, lethargy and hoarseness for 2 weeks. She was having intermittent episodes of left ear discharge and pain for 3 months with throbbing headache and vomiting episodes. She was previously treated for acute otitis externa.

She appeared comfortable under room air with stable vital signs. On otoscopic examination, the left ear canal was filled with pus, oedematous and there was a mass occupying the canal arising from the posterosuperior wall. The mass was smooth surfaced and didn't bleed on probing while the left tympanic membrane couldn't be visualised. The right ear was normal. Her intraoral examination revealed the curtain sign with left palatopharyngeal weakness. However, there was no trismus. There was a firm fullness felt on the left preauricular region measuring $7 \times 6 \mathrm{~cm}$ but there was no signs of inflammation. She also had left facial nerve palsy House-Brackmann Grade 2. Flexible nasopharyngolaryngoscopy showed pooling of saliva and the left vocal cord was immobile in paramedian position with a phonation gap consistent with left recurrent laryngeal nerve palsy. Her pure tone audiometry showed left moderate to profound mixed hearing loss and right mild to severe sensorineural hearing loss.

The total white blood cell count was raised up to $16.5 \times 10^{\%} / \mathrm{L}$. Both ESR and C-reactive protein were also raised with a reading of $123 \mathrm{~mm} / \mathrm{hr}$ and $40 \mathrm{mg} / \mathrm{L}$ respectively. She had electrolytes imbalance with hyponatraemia and hyperkalemia secondary to her poor oral intake.

She was admitted for initiation of intravenous antibiotics, corrections of electrolytes and dietary optimization. She was started on ryles' tube feeding and was referred to the Endocrine team to manage her diabetic status.

This lady had underlying diabetis mellitus, on insulin regime with a diabetic foot ulcer and hypertension. Her foot ulcer was fairly clean and was dressed daily. Her sugar control in the ward was below the range of $10 \mathrm{mmol} / \mathrm{L}$ but her $\mathrm{HbAlc}$ showed poor sugar control with reading of $14.5 \%$.

Swab was obtained from the left ear for culture and sensitivity and it grew Pseudomonas Aeruginosa. Biopsy was taken from the left external ear canal mass and was later reported as inflammatory granulation tissue by the pathologist.

She was started on intravenous ceftriaxone but once the culture grew Pseudomonas Aeruginosa, her antibiotics were upgraded to meropenem for better blood brain barrier penetration and was given for 2 -weeks. She was also given gentamicin with betamethasone ear drops.

She underwent high resolution computed tomography (HRCT) to see the extent of the disease. As shown on Figure 1, there was a soft tissue density in the left middle ear, part of the external ear canal and the left mastoid air cells. The ossicles and incudomalleolar joint were intact. Even though the first genu and the tympanic segment of the facial nerve were not well visualized, the rest of the facial nerve course were normal.

The HRCT also showed features of skull base osteomyelitis with abscesses in the left parapharyngeal extending to left fossa of rossenmuller, left torus tubarius, retropharyngeal, masticator space and left temporomandibular joint as shown in Figure 2.

There was an intracranial extension with meningeal enhancement in the left temporal region with bony erosions at the petrous apex, left anterior occipital condyle, squamous part of temporal bone, sphenoid bone, tegmen
Fig. 1 HRCT temporal, axial cuts showing soft tissue density in the left mastoid air cells (A). The ossicles were intact (B). The left facial nerve course was normal (C).
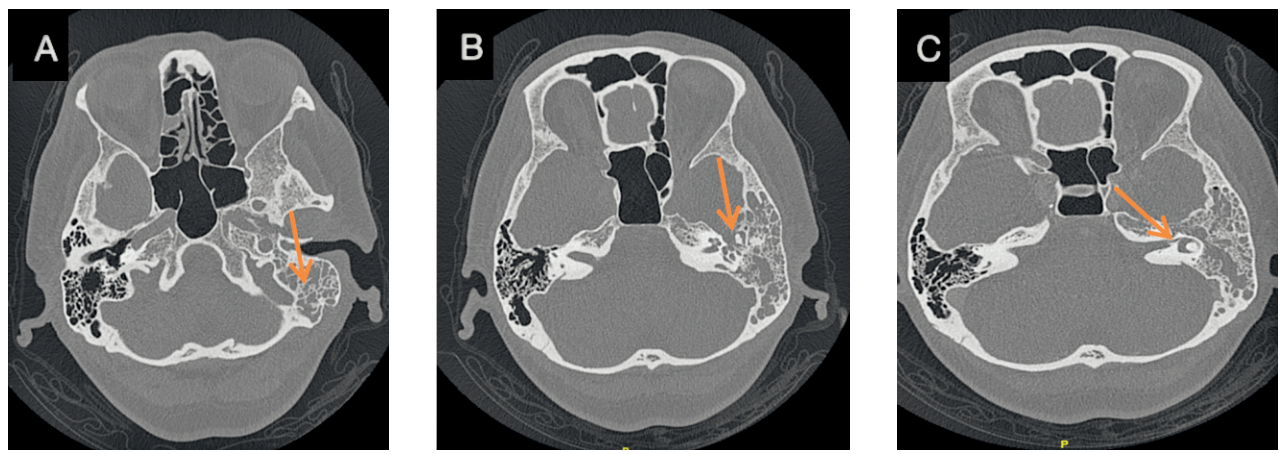


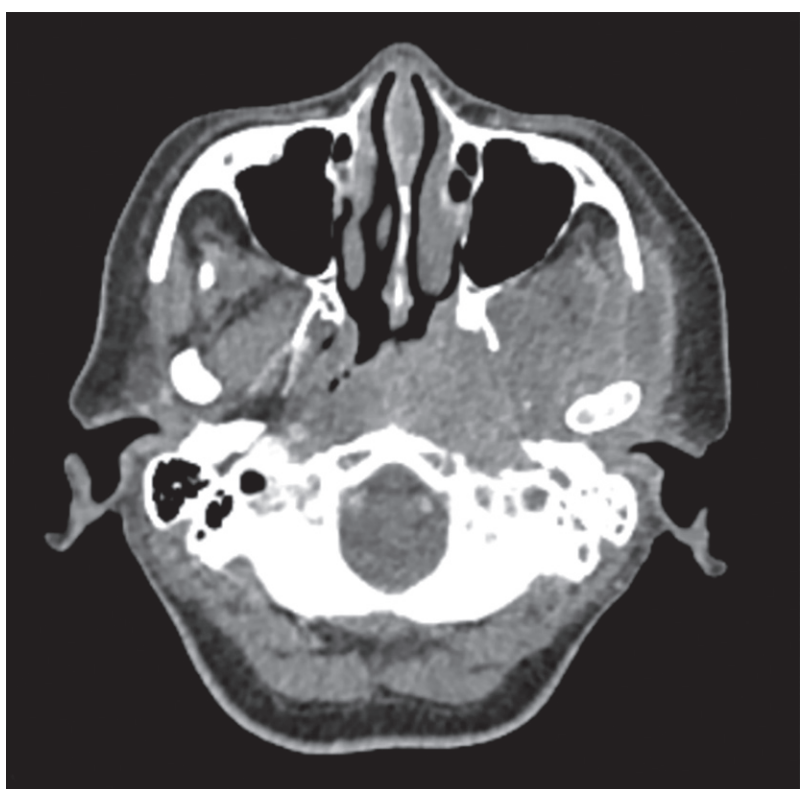

Fig. 2 HRCT temporal, axial cuts showing abscesses in the left parapharyngeal extending to left fossa of rossenmuller, left masticator space and left temporomandibular joint.

tympani, carotid canal, clivus and anterior part of foramen spinosum as shown in Figure 3.

After 2 weeks of intravenous meropenem, she was given oral ciprofloxacin for 4 weeks. Her symptoms improved as her otalgia and otorrhea resolved. Although her hoarseness persisted, she was well and was discharged home with oral ciprofloxacin.

She was seen again in clinic 2 weeks after and she was still pain free. Her ear symptoms such as otalgia and otorrhea improved. She was feeding via ryles tube with evident aspiration. However, there was a new finding of left hypoglossal nerve palsy showing significant muscle wasting of the left lateral tongue deviating to the left. The previously seen left facial nerve palsy reverted back to normal during examination. The other pre-existing left glossopharyngeal and left vagus nerve palsies showed no improvement. She was referred to the speech therapist for swallowing exercise. She was planned for a bone scan to monitor her disease progression and treatment response but patient refused for any further intervention.

\section{CASE REPORT 2}

The second case reported is a 53-year-old lady who presented with severe right ear otalgia for almost 5 months, throbbing in nature. She described it as a constant pain associated with right sided headache affecting her daily routine. The pain was relieved by oral analgesia but it only lasted a while. She also complaint of right ear fullness with tinnitus and reduced hearing. Otherwise she denied of any fever and there was no giddiness nor vertigo. The symptoms were not preceded by any respiratory infection or trauma.

She visited the local clinics multiple times and was given tropical ear drops and oral antibiotic but her symptoms were not relieved. She had underlying diabetic and hypertension, well controlled on oral medications and insulin injections.

She appeared comfortable under room air with no acute respiratory distress. There was right facial nerve palsy House-Brackmann Grade 2. On otoscopic examination, the right external ear canal was filled with a mass and the tympanic membrane could not be visualized; however the left ear canal was clear with intact tympanic membrane. She also had right glossopharyngeal and right vagus nerve palsies with curtain sign elicited intraorally and uvula deviated to the left. Her gag reflex was absent. The other cranial nerves examinations were unremarkable. There was a right level II lymph node measuring $2 \times 1 \mathrm{~cm}$, firm in consistency with well demarcated borders. Her pure tone audiometry showed right mild to severe mixed hearing loss and left mild to profound sloping sensorineural hearing loss.

She was admitted for intravenous ciprofloxacin given for 2 weeks, ofloxacin topical ear drops and pain control. During admission, her ESR was reported as $127 \mathrm{~mm}$ /hour and $\mathrm{C}$-reactive protein was positive with a reading of $48 \mathrm{mg} / \mathrm{L}$. Her total white blood cell count was $12.04 \times 10^{9} / \mathrm{L}$. A biopsy was obtained from the right ear canal mass reported as compatible with malignant otitis externa. Nasal endoscopy showed an enlarged right torus tubarius obliterating the right fossa of Rossenmuller. Biopsy of the right torus tubarius revealed no malignancy. Other findings were normal. Swab for culture and sensitivity from the right ear canal was reported as negative for any growth of microorganism.

Magnetic resonance imaging (MRI) was done towards the end of the 2 weeks of admission to look for any evidence of cholesteatoma or malignant lesions. As shown in Figure 4, there was a diffuse enhancing soft tissue lesion inferior to the right temporal lobe involving the right masticator space, right carotid space, right parapharyngeal space, pharyngeal mucosal space and retropharyngeal space. The lesion extended till the dural layer superiorly, inferiorly until the upper oropharynx and medially to the nasopharynx.
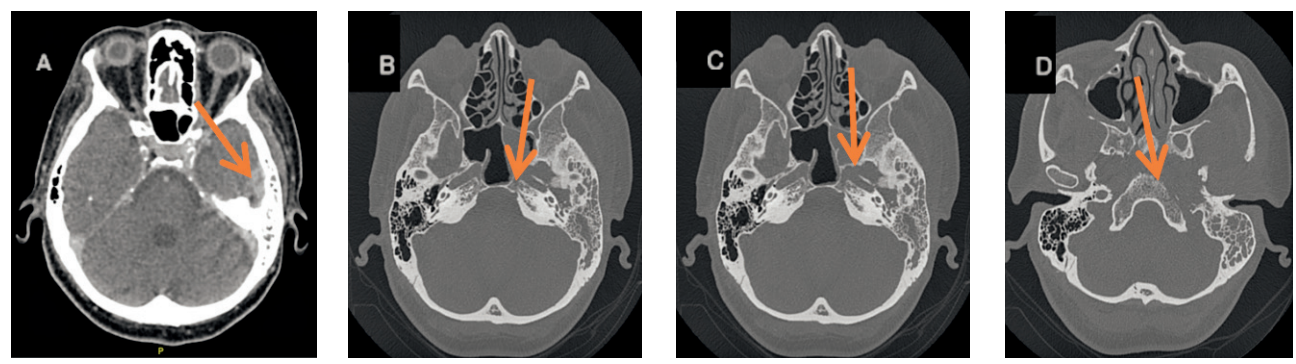

Fig. 3 HRCT temporal, axial cuts showing intracranial extension with meningeal enhancement in the left temporal region (A). Bony erosion were seen at left petrous apex (B), carotid canal (C) and clivus (D). 

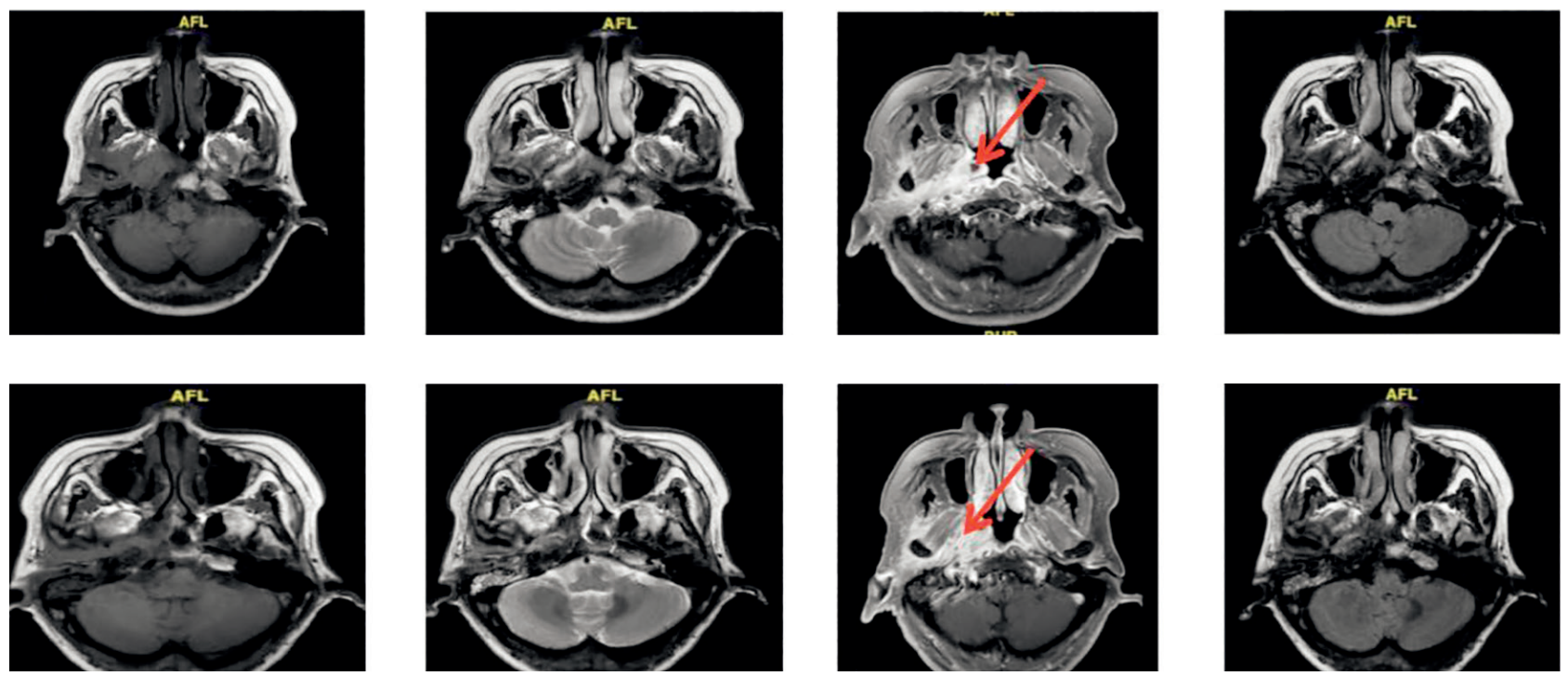

Fig. $4 \mathrm{MRI}$ images, axial cuts showing diffuse enhancing soft tissue lesion involving the right masticator, right parapharyngeal and retropharyngeal space.

She was given another 6 weeks of oral ciprofloxacin to complete 8 weeks all together. Her symptoms improved clinically with reducing pain and resolved ear discharge but she underwent right cortical mastoidectomy to obtain a tissue biopsy as we were still uncertain of her diagnosis. Intra-operatively there was sagging of the supero-posterior wall with intact tympanic membrane. When the tympanomeatal flap was raised, there was a soft tissue mass seen within the right mastoid antrum and right middle ear which was later reported as chronic inflammation. The middle ear mucosa appeared thickened with mucoid discharge.

Post operatively, she was doing well. Her pain was well controlled and there was no more ear discharge. There was improvement in the cranial nerve functions with right facial nerve House-Brackmann Grade 1 and uvula was central during intraoral examination. Her total white blood cell count dropped to $9.8 \times 10^{9} / \mathrm{L}$ and her ESR reading was decreasing to $111 \mathrm{~mm} /$ hour with a negative reading of C-reactive protein. Her pure tone audiometry also showed improvement with right moderate mixed hearing loss and left mild to profound sensorineural hearing loss.

\section{DISCUSSION}

Malignant otitis externa is a fulminant osteomyelitis involving the external auditory canal and the skull base following an episode of external ear infection. Being termed as a misnomer, it behaves aggresively like a malignancy. Patient in old age or patients with diabetis mellitus and debilitating condition are at higher risk for MOE (1).

Patients with MOE presents with severe otalgia and throbbing headaches just like the cases reported in this series. The intensity of pain is usually measured with the visual analogue scale (VAS). Both our patients had significant pain score according to the VAS. They usually show no improvement to local treatment and due to the ongoing infection, they may also have a discharging ear. The most commonly grown organism in MOE is Pseudomonas aeruginosa (95\%) (2) as reported in our first case report.

The otoscopic examination will reveal an edematous ear canal with granulation tissue at the osteocartilaginous junction. Besides the granulation tissue at the osteocartilaginous junction, there can also be a mass in the external ear canal. Biopsy of this mass is important to help to exclude other pathologies such as malignancies or cholesteatoma $(3,4)$ but most frequently, these biopsies obtained may not be significant (5) delaying the establishment of diagnosis.

MRI and CT scans are used to determine the anatomical extent of the disease and intracranial complications if any. Features of MOE on CT is reduce skull base density and bony erosions. Bony erosions of the skull base and petrous apex as seen in the first case report raises the suspicion of malignancy. In MOE, bony erosions on CT is only evident when at least $30 \%$ demineralisation of bone has happened which is usually in the later stage of the disease (6). MRI on the other hand determines the soft tissue changes, exact location and extent of the disease. MRI is highly sensitive but not so specific for MOE. The Technetium Tc 99 methylene diphosphonate bone scanning is the most useful imaging tool to evaluate the positive findings based on binding of the osteoblasts (3) but they may be positive in cases of malignancy as well (7).

As the disease progresses, patients can rarely present with cranial nerve involvement. Intracranial involvement can potentially alter the patient's mental status and even cause mortality. Cranial nerve involvement indicates a poor prognosis of the disease leading to death due to the complications $(8,9)$. The spread of infection from the external ear is through the fissures of Santorini and the osteocartilaginous junction. The lower cranial nerves are commonly affected in the skull base osteomyelitis as reported in both our cases. The other cause of lower cranial nerve palsy in these areas is the nasopharyngeal 
carcinoma (NPC) that is related to the posterolateral spread of the disease as well as the jugular foramen infiltration (10).

The facial nerve is the first and most common to be affected in MOE as it exits the stylomastoid foramen (7) and both our cases had facial nerve palsy on presentation. This is followed in order by the glossopharyngeal, vagus and accessory nerves as they exit the jugular foramen and the hypoglossal nerve as it exits the hypoglossal canal (11). The trigeminal and abducens nerve can be affected as well if there is petrous apex involvement. The spread of infection to the cavernous sinus and clivus may cause the olfactory, troclear and abducens nerve palsy (12). If the treatment is initiated early, the cranial nerve palsies in MOE can be reverted back to normal. Both our cases had facial nerve and lower cranial nerves involvement and they showed improvement after treatment suggesting MOE rather than malignancy.

Navin reported $43 \%$ of cranial neuropathies in a retrospective analysis of $23 \mathrm{MOE}$ cases where he mentioned that $60 \%$ cases were with facial nerve palsy, $30 \%$ with lower cranial nerves palsy in combinations of glossopharyngeal, vagus, accessory and hypoglossal nerve and 10\% had an extended cranial nerve palsies involving the facial nerve, lower cranial nerves and other cranial nerves. Out of the 10 cases reported, 4 died due to unrelated causes, 5 had resolution of disease but no cranial nerve palsy improvement and 1 required prolonged treatment of oral ciprofloxacin. And it was speculated that facial nerve palsy does not resolve after treatment because of its longer course of involvement compared to the others. This case series reported a recovery rate of $87 \%$ with $0 \%$ of mortality (13). In another journal, Ethan et al. reported that involvement of facial nerve palsy showed a significant extension of disease to the parapharyngeal and nasopharyngeal region in addition to the mastoid on computer tomography (4) just like the two cases reported in this series.

The choice of antibiotics for treatment is from the fluoroquinolone group namely ciprofloxacin that can attain high level of bone and soft tissue penentration, and third generation cephalosporins such as ceftazidime (2) and aminoglycosides such as gentamicin may be used in ciprofloxacin-resistant patients.

Surgical options are kept in view for local debridement, abscess drainage and bony sequestrum removal. Even though our patient in the first case was given intravenous meropenem for 2 weeks, she was then given a long 4 weeks course of oral ciprofloxacin and for our patient in the second case, she was treated with 8 weeks course of ciprofloxacin and they both responded well to the treatment.

There may be many instances where MOE was mistaken for NPC because both these diseases invades the bony structures aggressively. Both the cases in this series had imagings with involvement of fossa of rossenmuller mimicking NPC. Goh et al reported 14 cases with skull base osteomyelitis where the imagings showed a nasopharyngeal bulge involving the fossa of rossenmuller (14). He also mentioned that in MOE, the soft tissue enhancement is greater or equal to the mucosa and the spread of disease is along the fascial plane preserving the architecture whereas it was vice versa in NPC. The presence of abscesses in imagings should also lead to infection rather than malignancy.

Another significant distinguishing factor of MOE is the lateral structure involvements such as TMJ and parotid gland involvement. NPC usually does not extend laterally except in late cases due to perineural invasion or contiguous spread along the auriculotemporal nerve (15).

Clival involvement on MRI is shown for both MOE and malignancy (16). But for MOE specifically, the clival involvement is shown enhanced with marrow space hypointensity on $\mathrm{T} 1$ weighted images but hyperintense on $\mathrm{T} 2$ weighted images with enhancement of dura, parapharyngeal fat plane effacement and soft tissue mass in skull base (17). Ozgen et al reported the important function of diffusion-weighted sequence on MRI in differentiating MOE and malignancy (18). He said that malignant tumours such as NPC and lymphomas have very low apparent diffusion coefficients (ADC), while benign lesions such as MOE have higher ADC.

The raise of inflammatory markers such total white blood cell count, ESR and CRP should also lead the suspicion of infection rather than malignancy. MOE patients may have a reactive node but not in typical fashion like NPC. NPC patients commonly present with nodal metastasis starting from the retropharyngeal region extending to level II, III, IV, V and supraclavicular.

MOE may spread causing further complications such as lateral sinus thrombosis, internal jugular vein thrombosis, opthalmoplegia and blindness which can mimick malignancy. It is also important to correlate with the clinical history and examination where in MOE, patients commonly with underlying diabetes mellitus presents with ear pain and ear discharge with ear swabs growing Pseudomonas Aeruginosa.

\section{CONCLUSION}

Although MOE with cranial nerve involvement indicates extensive progression of the disease, it doesn't worsen the prognosis of the disease itself. In view of aggressive features of disease in imaging, it is important to differentiate MOE from malignancy as early intervention with long-term high dose antibiotics may lead to resolution of disease although the cranial nerve functions might not be reverted back to normal in all cases.

\section{REFERENCES}

1. Kaya İ, Sezgin B, Eraslan S, et al. Malignant Otitis Externa: A Retrospective Analysis and Treatment Outcomes. Turk Arch Otorhinolaryngl 2018; 56(2): 106.

2. Berenholz L, Katzenell U, Harell M. Evolving resistant pseudomonas to ciprofloxacin in malignant otitis externa. Laryngoscope 2002; 112(9): 1619-22.

3. Carfrae MJ, Kesser BW. Malignant otitis externa. Otolaryngol Clin North Am 2008; 41(3): 537-49.

4. Soudry E, Joshua BZ, Sulkes J, Nageris BI. Characteristics and prognosis of malignant external otitis with facial paralysis. Arch Otolaryngol Head Neck Surg 2007; 133(10): 1002-4.

5. Clark MP, Pretorius PM, Byren I, Milford CA. Central or atypical skull base osteomyelitis: diagnosis and treatment. Skull Base 2009; 19(4): 247. 
6. Hariga I, Mardassi A, Younes FB, et al. Necrotizing otitis externa: 19 cases'report. Eur Arch Otorhinolaryngol 2010; 267(8): 1193-8.

7. Ganhewa AD, Kuthubutheen J. A diagnostic dilemma of central skul base osteomyelitis mimicking neoplasia in a diabetic patient. BMJ Case Rep 2013; 2013: bcr2012007183.

8. Handzel O, Halperin D. Necrotizing (malignant) external otitis. Am Fam Physician 2003; 68(2): 309-12.

9. Jackson CG, von Doersten PG. The facial nerve: current trends in diagnosis, treatment, and rehabilitation. Med Clin North Am 1999; 83(1): 179-95.

10. Chong V, Fan Y. Hypoglossal nerve palsy in nasopharyngeal carcinoma. Eur Radiol 1998; 8(6): 939-45.

11. Amorosa L, Modugno GC, Pirodda A. Malignant external otitis: review and personal experience. Acta Otolaryngol 1996; 116(Supp 521): 3-16.

12. Grandis JR, Branstetter BF, Victor LY. The changing face of malignant (necrotising) external otitis: clinical, radiological, and anatomic correlations. Lancet Infect Dis 2004; 4(1): 34-9.
13. Mani N, Sudhoff H, Rajagopal S, Moffat D, Axon PR. Cranial nerve involvement in malignant external otitis: implications for clinical outcome. Laryngoscope 2007; 117(5): 907-10.

14. Goh J, Karandikar A, Loke S, Tan T. Skull base osteomyelitis secondary to malignant otitis externa mimicking advanced nasopharyngeal cancer: MR imaging features at initial presentation. Am J Otolaryngol 2017; 38(4): 466-71.

15. Schmalfuss IM, Tart RP, Mukherji S, Mancuso AA. Perineural tumor spread along the auriculotemporal nerve. American J Neuroradiol 2002; 23(2): 303-11.

16. Sreepada GS, Kwartler JA. Skull base osteomyelitis secondary to malignant otitis externa. Curr Opin Otolaryngol Head Neck Surg 2003; 11(5): 316-23.

17. Subburaman N, Chaurasia M. Skull base osteomyelitis interpreted as malignancy. J Laryngol Otol 1999; 113(8): 775-8.

18. Ozgen B, Oguz K, Cila A. Diffusion MR imaging features of skull base osteomyelitis compared with skull base malignancy. Am J Neuroradiol 2011; 32(1): 179-84. 